\title{
ON A HOMOMORPHISM BETWEEN GENERALIZED GROUP ALGEBRAS
}

\author{
BY ALVIN HAUSNER
}

Communicated by Edwin Hewitt, September 26, 1960

If $G=\{a, b, \cdots\}$ is a locally compact abelian group and $X$ $=\{x, y, \cdots\}$ a complex commutative Banach algebra, we denote by $B(G, X)$ the generalized group algebra in the sense of $[1 ; 2]$. An $X$-valued function $g$ defined over $G$ is in $B(G, X)$ if $g$ is strongly measurable and Bochner integrable with respect to Haar measure over $G$. We define $\|g\|_{B(G, X)}=\int_{G}|g(a)|_{X} d a$ and, with convolution as multiplication, $B(G, X)$ is a complex commutative $B$-algebra. In $[1$, p. 1606], it is shown that the space $\mathfrak{M}(B)$ of regular maximal ideals in $B(G, X)$ is homeomorphic with $\hat{G} \times \mathfrak{M}(X)$. Here, $\hat{G}=\{\hat{a}, \hat{b}, \cdots\}$ is the character group of $G$ and $\mathfrak{M}(X)$ denotes the space of regular maximal ideals in $X$, both in their usual topologies. If $\phi_{M}$ is the canonical homomorphism of $X$ onto the complex numbers associated with an $M \in \mathfrak{M}(X)$, then a function $g \in B(G, X)$ is represented on $\mathfrak{M}(B)$ by the function $\hat{g}(\hat{a}, M)=\int{ }_{G} \phi_{M} g(a)(a, \hat{a}) d a,[1$, p. 1604]. If $f \in L(G)$, $x \in X$, then $f x$ shall denote the function $(f x)(a)=f(a) x$ almost everywhere over $G$. Clearly $f x \in B(G, X)$. Further, finite linear combinations of functions of the type $f x$ with $f \in L(G), x \in X$ are dense in $B(G, X)$.

In this paper we propose to characterize the homomorphisms $T$ from $B(G, X)$ into $B\left(G, X^{\prime}\right)$ which are such that $T$ keeps $L(G)$ "pointwise invariant." More precise statements will be found in the theorems below.

We begin with

THEOREM 1. Let $G$ be a group such that $\hat{G}$ is connected and let $X$ and $X^{\prime}$ be commutative B-algebras with identities e, $e^{\prime}$ respectively. Suppose $\mathfrak{M}(X)$ is totally disconnected and $X^{\prime}$ is semi-simple. Let $T: B(G, X)$ $\rightarrow B\left(G, X^{\prime}\right)$ be a continuous homomorphism such that $T(f e)=f e^{\prime}$ for any $f \in L(G)$. Then there exists a continuous homomorphism $\sigma: X \rightarrow X^{\prime}$ such that $(T g)(a)=\sigma g(a)$ for any $g \in B(G, X)$.

Proof. ${ }^{1}$ If $g^{\prime} \in B\left(G, X^{\prime}\right)$ and $g^{\prime}$ is represented on its space of maximal ideals $\hat{G} \times \mathfrak{M}\left(X^{\prime}\right)$ as $\hat{f} \cdot \phi^{\prime}$ where $f \in L(G)$ and $\phi^{\prime}$ is a function defined on $\mathfrak{M}\left(X^{\prime}\right)$, then $g^{\prime}=f x^{\prime}$ for some $x^{\prime} \in X^{\prime}$. (Here, $\hat{f}(\hat{a})$ $=\int_{G} f(\hat{a})(a, \hat{a}) d a$. $)$ For, consider the function $F$ from $G$ to $X^{\prime}$ given

1 The author wishes to gratefully thank the referee of [1] for suggesting the following proof. 
by $F(a)=\int_{G} g^{\prime}(a)(a, \hat{a}) d a$. If $M^{\prime} \in \mathfrak{M}\left(X^{\prime}\right)$, then $\phi_{M^{\prime}}(F(\hat{a}))=\hat{f}(\hat{a}) \phi^{\prime}\left(M^{\prime}\right)$. If $f \neq 0$, there exists an $\hat{a}$ such that $\hat{f}(\hat{a}) \neq 0$. Let $x^{\prime}=F(\hat{a}) / \hat{f}(\hat{a})$ so that $\phi_{M^{\prime}}\left(x^{\prime}\right)=\phi^{\prime}\left(M^{\prime}\right)$. We see that $g^{\prime}$ and $f x^{\prime}$ are represented by the same function on $\hat{G} \times \mathfrak{M}\left(X^{\prime}\right)$. Now, since $X^{\prime}$ is semi-simple, $B\left(G, X^{\prime}\right)$ is semi-simple $\left[1\right.$, p. 1609] and thus $g^{\prime}=f x^{\prime}$.

For $\hat{a} \in \hat{G}, M^{\prime} \in \mathfrak{M}\left(X^{\prime}\right)$, we have in

$$
[T g]^{\sim}\left(\hat{a}, M^{\prime}\right)=\int_{G} \phi_{M^{\prime}}(T g)(a)(a, a) d a
$$

a continuous multiplicative linear functional on $B(G, X)$. This means that $[T g]^{\wedge}\left(\hat{a}, M^{\prime}\right)=\hat{g}\left(\tau(\hat{a}), \sigma^{*} M^{\prime}\right)$ for some $\tau(\hat{a}) \in \hat{G}, \sigma^{*} M^{\prime} \in \mathfrak{M}(X)$. $T$ thus induces a map $T^{*}: \hat{G} \times \mathfrak{M}\left(X^{\prime}\right) \rightarrow \hat{G} \times \mathfrak{M}(X)$ given by $T^{*}\left(\hat{a}, M^{\prime}\right)$ $=\left(\tau(\hat{a}), \sigma^{*} M^{\prime}\right)$. Since $\hat{G}$ is connected and $T^{*}$ is continuous, $T^{*}\left(\hat{G} \times\left\{M^{\prime}\right\}\right)$ is a connected set in $\hat{G} \times \mathfrak{M}(X)$ for each $M^{\prime} \in \mathfrak{M}\left(X^{\prime}\right)$. Since $\mathfrak{M}(X)$ is totally disconnected, $T^{*}\left(\hat{G} \times\left\{M^{\prime}\right\}\right) \subset \hat{G} \times\left\{\sigma^{*} M^{\prime}\right\}$. This is true because the connected components of $\hat{G} \times \mathfrak{M}(X)$ are precisely of the form $\hat{G} \times\{M\}$ with $M \in \mathfrak{M}(X)$. Since $T(f e)=f e^{\prime}, f \in L(G)$, we conclude that $\tau(\hat{a})=\hat{a}$ and $T^{*}$ is the product of the identity map on $\hat{G}$ and a map $\sigma^{*}: \mathfrak{M}\left(X^{\prime}\right) \rightarrow \mathfrak{M}(X)$.

Consider $f x \in B(G, X), f \in L(G)$. It gets represented as a product function on $\hat{G} \times \mathfrak{M}(X)$. From the nature of $T^{*}$ in the preceding paragraph, $T(f x)$ gets represented as a product function on $\hat{G} \times \mathfrak{M}\left(X^{\prime}\right)$ whose first factor is $\hat{f}(\hat{a})$. In view of the second paragraph of this proof, there exists $\sigma(x) \in X^{\prime}$ such that $T(f x)=\sigma(x) f$. The map $\sigma: X \rightarrow X^{\prime}$ is a continuous homomorphism as is easy to verify. As already remarked, finite linear combinations of functions $f x$ are dense in $B(G, X)$ and since $T$ is continuous, the theorem is proved.

In our next theorem $G$ will be taken compact and we require the following

Lemma. Suppose $G$ is a compact abelian group with Haar measure normalized to 1 , and $X$ is a complex commutative $B$-algebra with identity $e$ with no restrictions on $\mathfrak{M}(X)$. Let $\phi$ be a continuous homomorphism from $B(G, X)$ to $L(G)$ which is such that $\phi(f e)=f$ for all $f \in L(G)$. Then there exists an $M \in \mathfrak{M}(X)$ such that $(\phi g)(a)=\phi_{M} g(a)$ a.e. for any $g \in B(G, X)$.

Proof. Since $G$ is compact, the constant $X$-valued functions are in $B(G, X)$ and thus $X$ can be considered to be a subset of $B(G, X)$. In other words, if $x \in X$, we denote the function $f(a)=x$ (for almost all $a \in G)$ simply by $x$ itself. If $x, y \in X \subset B(G, X)$, then $x * y=\int_{G} x y d a$ $=x y$ since $m(G)=1$. (Here, $x y$ denotes the ordinary product of $x$ and $y$ in the $B$-algebra $X$.) Now $\phi$ is not identically zero on $X$ because 
$\phi(1 \cdot e)=1$. Further, for any $x \in X, \phi(x * e)=\phi(x e)=\phi(x)=\phi(x) * \phi(e)$ $=\phi(x) * 1=\int_{G} \phi(x)(a) d a=$ a constant a.e. over $G$. Hence each $x \in X$ is mapped by $\phi$ onto a constant function in $L(G) . \phi$ is additive on $X$ and furthermore: $\phi(x * y)=\phi(x y)=\phi(x) * \phi(y)=\phi(x) \phi(y)$ for any $x, y \in X$. Consequently $\phi$ is a continuous nonzero multiplicative linear functional on the $B$-algebra $X$ and, as such, there exists an $M \in \mathfrak{M}(X)$ with $\phi(x)=\phi_{M}(x)$ for $x \in X$.

Choose an arbitrary $f \in L(G), \quad x \in X$ and $\hat{a} \in \hat{G}$. We have: $\phi\left(f x *(\cdot, \hat{a})^{-1} e\right)=\phi\left(\hat{f}(\hat{a})(\cdot, \hat{a})^{-1} x\right)=\hat{f}(\hat{a}) \phi\left((\cdot, \hat{a})^{-1} x\right)=\phi(f x) * \phi\left((\cdot, \hat{a})^{-1} e\right)$ $=\phi(f x) *(\cdot, a)^{-1}=(\cdot, \hat{a})^{-1}[\phi(f x)]^{-}(\hat{a})$. Hence, for each $\hat{a} \in \hat{G}$, $[\phi(f x)]^{\wedge}(\hat{a})=(a, \hat{a}) \hat{f}(\hat{a}) \phi\left((a, \hat{a})^{-1} x\right)$ for all $a \in G$ except possibly over a set of measure 0 . We can therefore choose an $a_{0}$ (depending on $\hat{a}$ ) for which the last equation is true and substituting we find: $[\phi(f x)]^{\mathcal{C}}(\hat{a})=\left(a_{0}, \quad \hat{a}\right) \hat{f}(\hat{a}) \phi\left(\left(a_{0}, \quad \hat{a}\right)^{-1} x\right)=\left(a_{0}, \quad \hat{a}\right)\left(a_{0}, \quad \hat{a}\right)^{-1} \hat{f}(\hat{a}) \phi(x)$ $=\hat{f}(\hat{a}) \phi_{M}(x)$. This implies $\phi(f x)=\phi_{M}(x) f$ for all $f \in L(G), x \in X$.

Taking finite linear combinations of functions of the type $f x$ with $f \in L(G)$ and $x \in X$, we can find a sequence $\left\{f_{n}\right\}$ such that $f_{n} \rightarrow g$ for any $g \in B(G, X)$ with $\phi\left(f_{n}\right)=\phi_{M}\left(f_{n}\right)$. Hence $\phi(g)=\phi_{M}(g)$ since $\phi$ is continuous and the lemma is established.

Theorem 2. Let $G$ and $X$ be as in the lemma and let $X^{\prime}$ denote a semi-simple B-algebra with identity $e^{\prime}$. Suppose $T: B(G, X) \rightarrow B\left(G, X^{\prime}\right)$ is a continuous homomorphism such that $T(f e)=f e^{\prime}$ for any $f \in L(G)$. Then there exists a continuous homomorphism $\sigma: X \rightarrow X^{\prime}$ such that $(T g)(a)=\sigma g(a)$ for any $g \in B(G, X)$.

Proof. Let $\{W\}$ be the set of neighborhoods of the identity $0 \in G$ and let $\left\{j_{W}\right\}$ be an approximate identity in $L(G)$. Then, if $f \in L(G)$, $x \in X$, we have $T\left(j_{W} * f x\right)=T\left(j_{W} x\right) * f e^{\prime} \rightarrow T(f x)$ as $W \rightarrow 0$. Taking Fourier transforms we find $\left[T\left(j_{W} x\right)\right]^{\wedge}\left(f e^{\prime}\right)^{\wedge} \rightarrow[T(f x)]^{\wedge}$ so that $\left[T\left(j_{W} x\right)\right]^{\wedge}(\hat{a})$ converges as $W \rightarrow 0$ for each $\hat{a} \in \hat{G}$. Call this limit $\sigma_{\hat{a}}(x)$. It is clear that $\sigma_{\theta}(x)$ is independent of the approximate identity $\left\{j_{W}\right\}$ and the function $f$ defining it. We have $[T(f x)]^{\wedge}(\hat{a})=\sigma_{\hat{a}}(x) \hat{f}(\hat{a}) e^{\prime}$.

Let $M^{\prime} \in \mathfrak{M}\left(X^{\prime}\right)$ and consider the map $\phi_{M^{\prime}} \circ T$ from $B(G, X)$ to $L(G)$. If $f e \in B(G, X)$ with $f \in L(G)$, then $\left(\phi_{M^{\prime}} \circ T\right)(f e)=\phi_{M^{\prime}}\left(f e^{\prime}\right)=f$. The map $\phi_{M^{\prime}} \circ T$ is continuous and the lemma applies to it. Therefore, there is an $M \in \mathfrak{M}(X)$, depending on $M^{\prime} \in \mathfrak{M}\left(X^{\prime}\right)$, such that $\phi_{M^{\prime}} \circ T=\phi_{M}$. Now: $\phi_{M^{\prime}}[T(f x)]^{\wedge}(\hat{a})=\left[\left(\phi_{M^{\prime}} \circ T\right)(f x)\right]^{\frown}(\hat{a})=\hat{f}(\hat{a}) \phi_{M}(x)$ $=\hat{f}(\hat{a}) \phi_{M^{\prime}}\left(\sigma_{\hat{a}}(x)\right)$. This means $\phi_{M^{\prime}}\left(\sigma_{\hat{d}}(x)\right)=\phi_{M}(x)$ for each $M^{\prime} \in \mathfrak{M}\left(X^{\prime}\right)$ and each $\hat{a} \in \hat{G}, x \in X$.

We show that $\sigma_{\hat{a}}(x)$ is actually independent of $a$. Suppose $\sigma_{a_{1}}(x)$ $=y_{1}, \sigma_{a_{2}}(x)=y_{2}$ and $\hat{a}_{1} \neq \hat{a}_{2}$. For any $M^{\prime} \in \mathfrak{M}\left(X^{\prime}\right)$ we have $\phi_{M^{\prime}}\left(y_{1}\right)$ $=\phi_{M^{\prime}}\left(y_{2}\right)=\phi_{M}(x)$. Since $X^{\prime}$ is semi-simple, we must have $y_{1}=y_{2}$ and 
so $\sigma_{a}(x)$ is independent of $\hat{a}$. Write $\sigma_{a}(x)=\sigma(x), \sigma$ is a continuous homomorphism from $X$ to $X^{\prime}$. We have, consequently, shown that $[T(f x)]^{\wedge}(\hat{a})=\sigma(x) \hat{f}(\hat{a})$ and this means $T(f x)=\sigma(x) f$ for all $f \in L(G)$, $x \in X$, because $B\left(G, X^{\prime}\right)$ is semi-simple if $X^{\prime}$ is semi-simple. Continuing in a manner like that at the end of the lemma or the end of Theorem 1, we find that $(T g)(a)=\sigma g(a)$ for all $g \in B(G, X)$. This completes the proof.

We remark that, conversely, if $\sigma: X \rightarrow X^{\prime}$ is a continuous homomorphism, then the map $(T g)(a)=\sigma g(a)$ from $B(G, X)$ to $B\left(G, X^{\prime}\right)$ is a continuous homomorphism with no restrictions on $G, \hat{G}, X$ or $X^{\prime}$. The proof is easy and is omitted.

THEOREM 3. In either Theorem 1 or 2, if $T$ is an isomorphism from $B(G, X)$ onto $B\left(G, X^{\prime}\right)$, then $\sigma$ is an isomorphism from $X$ onto $X^{\prime}$.

Proof. $\sigma$ is one-one for if $x_{1} \neq x_{2}, x_{1}, x_{2} \in X$, then $f x_{1} \neq f x_{2}$ where $f \in L(G), f \neq 0$. Since $T$ is one-one, $T\left(f x_{1}\right)=\sigma\left(x_{1}\right) f \neq T\left(f x_{2}\right)=\sigma\left(x_{2}\right) f$ so that $\sigma\left(x_{1}\right) \neq \sigma\left(x_{2}\right) . \sigma$ is onto $X^{\prime}$, for choose any $x^{\prime} \in X^{\prime}$. Find an $f \in L(G)$ such that $\hat{f}(\hat{0}) \neq 0$. Since $T$ is onto, there is a $g \in B(G, X)$ such that $\sigma g=f x^{\prime}$. Taking Fourier transforms: $[\sigma g]^{\frown}(\hat{a})=\sigma \hat{g}(\hat{a})$ $=\hat{f}(\hat{a}) x^{\prime}$. Setting $\hat{a}=\hat{0}$, we find $\sigma \hat{g}(\hat{0})=\hat{f}(\hat{0}) x^{\prime}$ so that $\sigma(\hat{g}(\hat{0}) / \hat{f}(\hat{0}))=x^{\prime}$. Hence, there is an $x=\hat{g}(\hat{0}) / \hat{f}(\hat{0}) \in X$ such that $\sigma(x)=x^{\prime}$.

\section{REFERENCES}

1. Alvin Hausner, The tauberian theorem for group algebras of vector-valued functions, Pacific J. Math. vol. 7 (1957) pp. 1603-1610.

2. - On generalized group algebras, Proc. Amer. Math. Soc. vol. 10 (1959) pp. 1-10.

The City College of New York 